\title{
DESCRIPCIÓN ANALÍTICA DE LA RESISTENCIA DEL SUELO A LA PENETRACIÓN
}

\author{
Pedro Hurtado de Mendoza Borges ${ }^{1}$, Hélio Garcia Leite ${ }^{2}$, Haroldo Carlos Fernándes ${ }^{3}$, Frederico Cássio Moreira \\ Martins ${ }^{4}$, Zaíra Morais dos Santos Hurtado de Mendoza ${ }^{5}$
}

\begin{abstract}
RESUMEN
Apesar de no existir un criterio uniforme para evaluar la compactación del terreno, la mayoría de los autores recomiendan la resistencia del suelo a la penetración como principal indicativo. En este trabajo se formularon modelos estadísticos para expresar la referida resistencia, en función de la profundidad y del contenido de agua en el suelo, con base en datos experimentales. El suelo fue clasificado como ferralítico amarillo, sometido a la siembra directa durante los últimos cinco años con los cultivos de maíz y frijol. Se utilizó el penetrometro electrónico PNT 2000. Los datos fueron coleccionados para tres contenidos de agua en el suelo (aproximadamente 20, 32 y 40\%) y la profundidad varió entre 0 y $0,60 \mathrm{~m}$. En el procesamiento de los datos fueron utilizados los programas computacionales STATISTICA 8.0 y CurveExpert 1.4. Los modelos más apropiados se seleccionaron de acuerdo con el coeficiente de determinación, los desvíos entre valores observados y estimados y la magnitud de los residuos. Se concluyó que la resistencia del suelo a la penetración puede ser descrita analíticamente usándose modelos no lineares y que estos pueden ser una útil herramienta para inferir sobre la compactación del suelo, en función del contenido de agua.
\end{abstract}

Palabras-claves: compactación del suelo, modelos no lineares, siembra directa.

\section{ABSTRACT \\ ANALYTIC DESCRIPTION OF THE SOIL RESISTANCE TO THE PENETRATION}

Despite the absence of uniform criteria for evaluating soil compaction most authors recommend the soil resistance to the penetration as the main indication. This work aimed to develop statistical models to express the mentioned resistance, depending on the depth and soil moisture by means of experimental data. The soil was classified as yellow ferralitic, under no-tillage in the last five years with maize and beans. It was used an electronic penetrometer of brand PNT 2000. The data were collected in three moisture levels (about 20\%, 32\% and 40\%) with the depth ranging from 0 to $0.60 \mathrm{~m}$. For the data processing it was used softwares CurveExpert 1.4 and STATISTICA 8.0. The most appropriate models were selected according to the coefficient of determination, the deviations between observed and predicted values and the magnitude of the waste. It was concluded that the soil resistance to the penetration can be described analytically by non-linear models and these can be a useful tool to infer soil compaction depending on soil moisture.

Keywords: soil compaction, nonlinear models, no-tillage system.

\footnotetext{
Recebido para publicação em 13/09/2011. Aprovado em 22/10/2012.

1 - Ingeniero Agrícola, Profesor Adjunto de la UFMT/Cuiabá - MT, e-mail: pborges@ufmt.br

2 - Ingeniero Forestal, Profesor Asociado de la UFV, Viçosa-MG.

3 - Ingeniero Agrícola, Profesor Asociado de la UFV, Viçosa-MG.

4 - Ingeniero Agrónomo, Doctorando, Departamento de Ingeniería Agrícola, UFV, Viçosa-MG.

5 - Ingeniera Forestal, Profesora Adjunta de la UFMT, Cuiabá-MT.
} 


\section{INTRODUCCIÓN}

El estado de compactación del suelo tiene una importancia extraordinaria en el planeamiento de las operaciones que se deben realizar durante la implantación de una cultura agrícola, pues la selección de los equipos para la preparación y el manejo del terreno está directamente relacionada con el referido factor. Apesar de no existir un criterio uniforme para evaluar la compactación del terreno, la resistencia del suelo a la penetración ha sido utilizada frecuentemente como principal indicativo (BEUTLER \& CENTURION, 2004; BEUTLER et al., 2006; MOLIN et al., 2006; PÉREZ, 2006; ACOSTA, 2008; BONNIN et al., 2010).

Los implementos utilizados para preparar el suelo actúan a diferentes profundidades y de distinta forma. Así, los correspondientes al sistema convencional como son el arado y las gradas, revuelven mucho el terreno, principalmente en la camada de 0 a $25 \mathrm{~cm}$. Por otro lado, los sistemas conservacionistas son caracterizados por el poco o ningún revolvimiento del suelo, como son el preparo mínimo o reducido y la siembra directa, respectivamente. En este sentido Tormena et al. (2002) y Fabrizzi et al. (2005), concluyeron que el sistema de manejo puede interferir en el contenido de agua y en la resistencia del suelo a la penetración.

Con el aumento del contenido de agua, disminuye la acción de las fuerzas de cohesión entre las partículas del suelo y el interno, ocasionando, entonces, la disminución de la resistencia del suelo a la penetración (CUNHA et al., 2002; ACOSTA, 2008). Assis et al. (2009) estudiaron la mencionada resistencia para diferentes contenidos de agua y tipos de suelos, sin embargo no desarrollaron ecuaciones empíricas entre esas variables. También, Bonnin et al. (2010) confirmaron una elevada correlación entre la resistencia del suelo a la penetración y la profundidad, no obstante, esa dependencia no fue descrita analíticamente.

En la bibliografía consultada se verifica que la resistencia del suelo es influenciada por el sistema de preparo y consecuentemente por el contenido de agua, variando con la profundidad. Con base en esos trabajos se formuló la hipótesis de que, independientemente del tipo de solo y contenido de agua, la referida resistencia aumenta con la profundidad hasta un punto máximo, después decrece en determinado intervalo y finalmente presenta un comportamiento asintótico. Esa hipótesis motivó la realización de este trabajo que tiene como objetivo desarrollar un modelo estadístico para relacionar la resistencia mecánica del suelo a la penetración, en función de la profundidad.

\section{MATERIAL Y MÉTODOS}

El experimento se realizó durante dos años consecutivos, en un área perteneciente a la Universidad Federal de Viçosa, situada en la ciudad de Viçosa/MG, definida por las coordenadas geográficas $20^{\circ} 45^{\prime} 14$ " de latitud Sur y $42^{\circ} 52^{\prime}$ 53 " de longitud Oeste y altitud media de $650 \mathrm{~m}$. En la región predomina el relevo montañoso (aproximadamente 85\%). El clima fue clasificado como Cwa, templado húmedo con veranos calientes e inviernos secos (KÖPPEN \& GEIGER, 1928). La temperatura anual media varia entre $14,0{ }^{\circ} \mathrm{C}$ e 26,1 ${ }^{\circ} \mathrm{C}$. El terreno fue sometido a la siembra directa durante los últimos cinco años con los cultivos de maíz y frijol de forma alternada.

Tomándose como referencia los trabajos de Alves \& Seraphin (2004) y de Grego \& Vieira (2005), se seleccionó un área de $1600 \mathrm{~m}^{2}(40$ x 40 m) para el muestreo en una malla cuadrada de 64 puntos, separados en $5 \mathrm{~m}$ de lado. En cada punto se determinó la resistencia del suelo a la penetración, utilizándose el penetrometro, modelo PNT 2000, fabricado por la DLG, siguiéndose la norma ASAE S 313.3 (ASABE, 2006). Ese procedimiento fue realizado en cada año para tres periodos definidos, tales como: húmedo (enero, febrero, noviembre y diciembre), intermedio (marzo, abril, septiembre y octubre) y seco (mayo, junio, julio y agosto).

Para el desarrollo de los modelos se consideró la media de la resistencia del suelo a la penetración a cada $25 \mathrm{~mm}$ de profundidad, con base en los datos provenientes de 128 puntos (64 por año) coleccionados en los tres períodos. Luego, la expresión general se definió como:

$R_{\text {sp }}=f(p)$

en que

$\mathrm{R}_{\mathrm{sp}}=$ Resistencia del suelo a la penetración (MPa); $\mathrm{p}=$ Profundidad $(\mathrm{mm})$. 
Se ajustaron diversos modelos de regresión no lineal, utilizándose las herramientas disponibles en los programas computacionales CurveExpert 1.4 y STATISTICA 8.0. Las ecuaciones empíricas relacionan la resistencia del suelo a la penetración para cada período del año, en función de la profundidad. El ajuste de los modelos propuestos fue evaluado analíticamente, de acuerdo con el coeficiente de determinación $\left(\mathrm{R}^{2}\right)$ y la Prueba $t$ de Student (MONTGOMERY et al., 2004; LEVINE et al., 2005; WEISBERG, 2005). Los modelos se comprobaron también, elaborándose el gráfico de valores estimados en función de los observados, el gráfico de residuos y el histograma de frecuencias de los residuos.

La independencia de los residuos o inexistencia de auto correlación es una premisa básica para os modelos desarrollados, pues la colección de los datos varió con el tiempo. Esta condición fue analizada de acuerdo con el gráfico de residuos en función del tiempo de colección de los datos (período del año) y verificada analíticamente conforme la estadística de Durbin-Watson (LEVINE et al., 2005).

\section{RESULTADOS Y DISCUSIÓN}

El suelo se clasificó como Latosuelo rojo-amarillo de textura franco-argilosa (EMBRAPA, 1999). Considerándose los dos años de la investigación, los valores del contenido de agua (base volumétrica) variaron de 35 a $45 \%$, de 25 a $35 \%$ y de 15 a $25 \%$, respectivamente, para los períodos definidos como húmedo, intermedio y seco. En el Cuadro 1 se presentan los valores medios de las variables utilizadas para caracterizar el área experimental.

Considerándose los resultados de los procedimientos analíticos y gráficos, se optó por la relación entre el modelo de densidad (Harris) y la función exponencial asociada. La referida dependencia funcional tiene las características principales siguientes: inicio en el origen del sistema de coordenadas, aumento de la resistencia a la penetración con el incremento de la profundidad hasta alcanzar un valor máximo, disminución de la resistencia con el aumento de la profundidad y definición de un valor asintótico. Esa tendencia concuerda con la distribución de los datos experimentales (Figuras 1, 2 y 3).

Cuadro 1. Valores medios de masa seca de la cobertura vegetal e de los atributos físicos del suelo en el área experimental

\begin{tabular}{|c|c|c|}
\hline \multicolumn{2}{|c|}{ Variable y unidad } & Valor \\
\hline \multicolumn{2}{|l|}{ Masa seca de la cobertura vegetal $\left(\mathrm{t} \mathrm{ha}^{-1}\right)$} & 5,59 \\
\hline \multicolumn{2}{|l|}{ Masa específica del suelo $\left(\mathrm{g} \mathrm{cm}^{-3}\right)$} & 1,53 \\
\hline \multirow{3}{*}{$\begin{array}{l}\text { Contenido de agua en el suelo } \\
\qquad(\% \mathrm{bv})\end{array}$} & Período húmedo & 42,78 \\
\hline & Período intermedio & 28,62 \\
\hline & Período seco & 19,97 \\
\hline \multirow{3}{*}{$\begin{array}{c}\text { Composición granulométrica } \\
(\%)\end{array}$} & Macro porosidad & 12,83 \\
\hline & Micro porosidad & 38,64 \\
\hline & Porosidad total & 51,47 \\
\hline \multirow{4}{*}{$\begin{array}{l}\text { Textura del suelo } \\
(\%)\end{array}$} & Argila & 48,06 \\
\hline & Limo & 26,93 \\
\hline & Arena gruesa & 7,19 \\
\hline & Arena fina & 17,82 \\
\hline
\end{tabular}




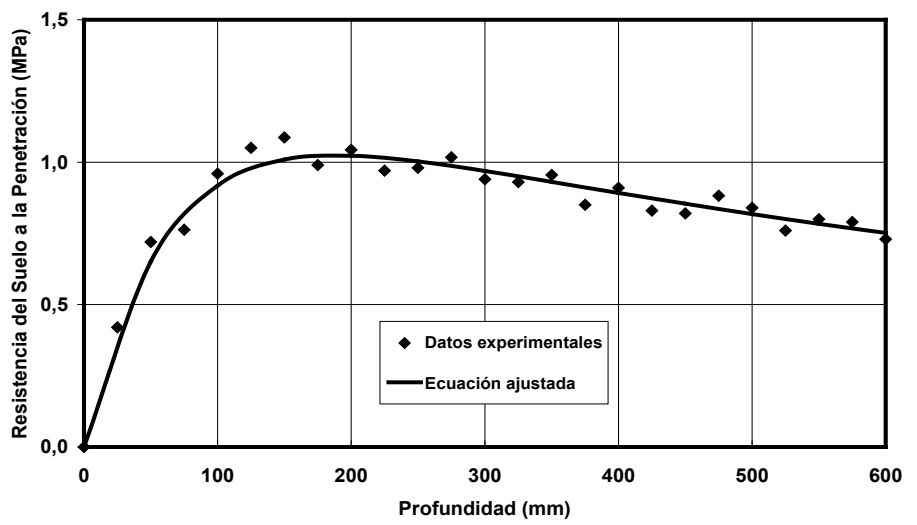

Figura 1. Diagrama de dispersión con los datos experimentales y curva de la ecuación ajustada para el período húmedo.

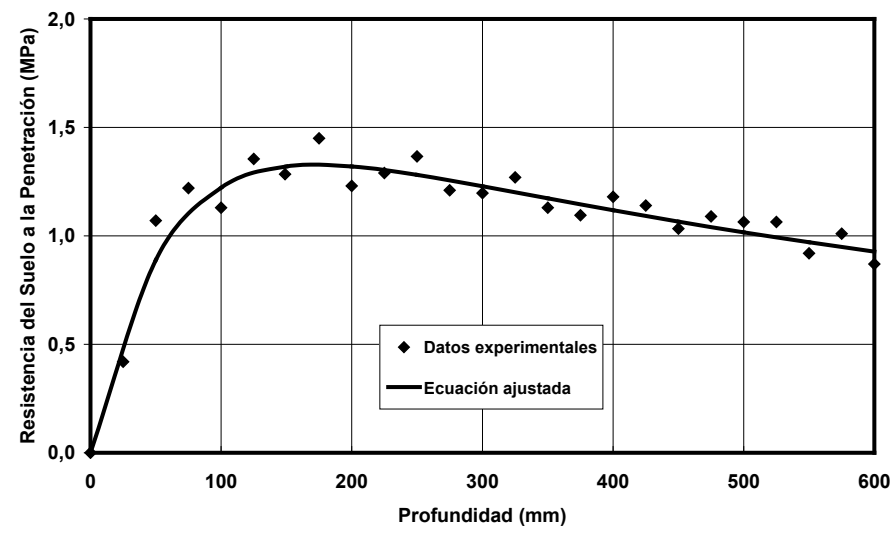

Figura 2. Diagrama de dispersión con los datos experimentales y curva de la ecuación ajustada para el período intermediario.

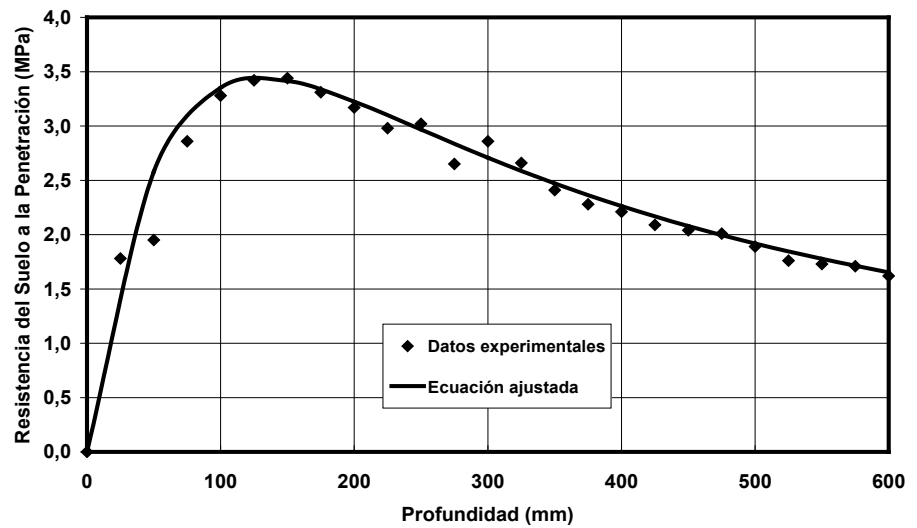

Figura 3. Diagrama de dispersión con los datos experimentales y curva de la ecuación ajustada para el período seco. 
En las Figuras 1, 2 y 3 se puede observar que, independiente del período del año, la resistencia del suelo a la penetración aumenta rápidamente hasta la profundidad de $100 \mathrm{~mm}$, alcanza el punto máximo entre 100 y $200 \mathrm{~mm}$, después decrece gradualmente hasta próximo de $400 \mathrm{~mm}$ y finaliza con tendencia asintótica a partir de los $400 \mathrm{~mm}$. Ese comportamiento fue semejante al obtenido por Assis \& Lanças (2005), Santos et al. (2005), Carvalho et al. (2006), Weirich Neto et al. (2006), Cunha et al. (2009) y Assis et al. (2009). También, la proximidad de la curva ajustada a los datos experimentales indica que los modelos elaborados pueden ser apropiados para estimar la resistencia del suelo a la penetración, en función de la profundidad, considerándose el período del año.

Debemos destacar que para el intervalo de profundidad comprendido entre 100 y 200 $\mathrm{mm}$, con base en las Figuras 1, 2 y 3 se puede inferir que los valores de resistencia del suelo a la penetración variaron de 1,0 a 3,5 $\mathrm{MPa}$. Esos limites incluyen los rangos de valores obtenidos por Beutler et al. (2006) y Weirich et al. (2006), respectivamente, de 2,0 a 3,0 $\mathrm{MPa}$ y de 2,0 a 2,2 MPa. También, el limite superior de nuestro trabajo está próximo al determinado por Assis \&
Lanças (2005) de 3,39 MPa para esa profundidad en condiciones experimentales semejantes y fue superior a los estimados por Carvalho et al. (2006) de 2,8 MPa y Cunha et al. (2009) de 2,6 MPa. Sin embargo, el limite superior de esta investigación se comportó de manera diferente cuando se comparó con los resultados presentados por Assis et al. (2009), es decir fue mayor que 2,0 $\mathrm{MPa}$ y menor que 4,5 $\mathrm{MPa}$, para los menores y mayores contenidos de agua, respectivamente. De todas formas, la proximidad de los valores estimados por medio de las curvas a los determinados experimentalmente, tanto en este como en otros estudios, confiere adecuada confiabilidad a los modelos elaborados.

En el Cuadro 2 se pueden observar las ecuaciones ajustadas y los correspondientes coeficientes de determinación $\left(\mathrm{R}^{2}\right)$ para cada período del año, considerándose el contenido de agua en el suelo. Los coeficientes de determinación de esta investigación fueron superiores al valor mínimo de 0,70 sugerido por Montgomery et al. (2004), Levine et al. (2005), Weisberg (2005) para ajustes de curvas, aunque este no es el único criterio adoptado en el presente trabajo para definir la confiabilidad de los modelos propuestos.

Cuadro 2. Ecuaciones ajustadas para la resistencia del suelo a la penetración, en función de la profundidad y coeficientes de determinación $\left(\mathrm{R}^{2}\right)$ en cada período del año

\begin{tabular}{|c|c|c|}
\hline $\begin{array}{c}\text { Período del año } \\
\text { (Contenido de agua) }\end{array}$ & Equação ajustada & $\mathrm{R}^{2}$ \\
\hline $\begin{array}{c}\text { Húmedo (enero, febrero, } \\
\text { noviembre y diciembre ) }\end{array}$ & $\mathrm{R}_{\mathrm{sp}}=\frac{0,8846\left(1-\mathrm{e}^{-0,0151846 \cdot \mathrm{p}}\right)}{0,700852+0,0001822 \cdot \mathrm{p}^{1,2301}}$ & 0,75 \\
\hline $\begin{array}{c}\text { Intermedio (marzo, abril, } \\
\text { septiembre y octubre) }\end{array}$ & $\mathrm{R}_{\mathrm{sp}}=\frac{0,9473\left(1-\mathrm{e}^{-0,0163469 \cdot \mathrm{p}}\right)}{0,574732+0,00017142 \cdot \mathrm{p}^{1,2295}}$ & 0,72 \\
\hline $\begin{array}{c}\text { Seco (mayo, junio, julio y } \\
\text { agosto) }\end{array}$ & $\mathrm{R}_{\mathrm{sp}}=\frac{0,9885\left(1-\mathrm{e}^{-0,0182679 \cdot \mathrm{p}}\right)}{0,184236+0,0001628 \cdot \mathrm{p}^{1,2263}}$ & 0,78 \\
\hline
\end{tabular}


De acuerdo con la Prueba t de Student, los valores teóricos y experimentales no presentaron diferencias significativas (Cuadro 3). Luego, se puede aceptar la hipótesis de que los datos observados y los determinados por los modelos son equivalentes. Con base en esa condición se puede afirmar que las ecuaciones obtenidas son apropiadas para describir la resistencia del suelo a la penetración, en función de la profundidad.

Para evaluar la relación entre los valores de la resistencia del suelo a la penetración determinados experimentalmente y los estimados por los modelos fueron elaborados los diagramas de dispersión de las Figuras 4, 5 y 6. En esas Figuras se puede constatar que los puntos están distribuidos encima y debajo de la bisectriz, independientemente del contenido de agua en el suelo. También, se puede observar que la mayoría de los puntos se encuentran próximos de la bisectriz, lo que indica poca diferencia entre los valores teóricos y reales.

Cuadro 3. Resultados de la Prueba t de Student para los tres períodos del año

\begin{tabular}{|c|c|c|c|c|}
\hline \multirow{2}{*}{ Período del año } & \multicolumn{2}{|c|}{ Valores de $\mathrm{t}$} & \multirow{2}{*}{ Significancia } \\
\cline { 2 - 3 } & \multirow{2}{*}{ Calculado } & $\alpha=5 \%$ & $\alpha=1 \%$ & \\
& 0,0555 & & & $\mathrm{n}$ \\
\hline Húmedo & 0,1418 & \multirow{2}{*}{1,6772} & 2,4066 & $\mathrm{~ns}$ \\
\hline Intermedio & 0,2481 & & & $\mathrm{~ns}$ \\
\hline Seco & & & \\
\hline
\end{tabular}

Obs. $\alpha$ : Nivel de probabilidad; ns: no significativo

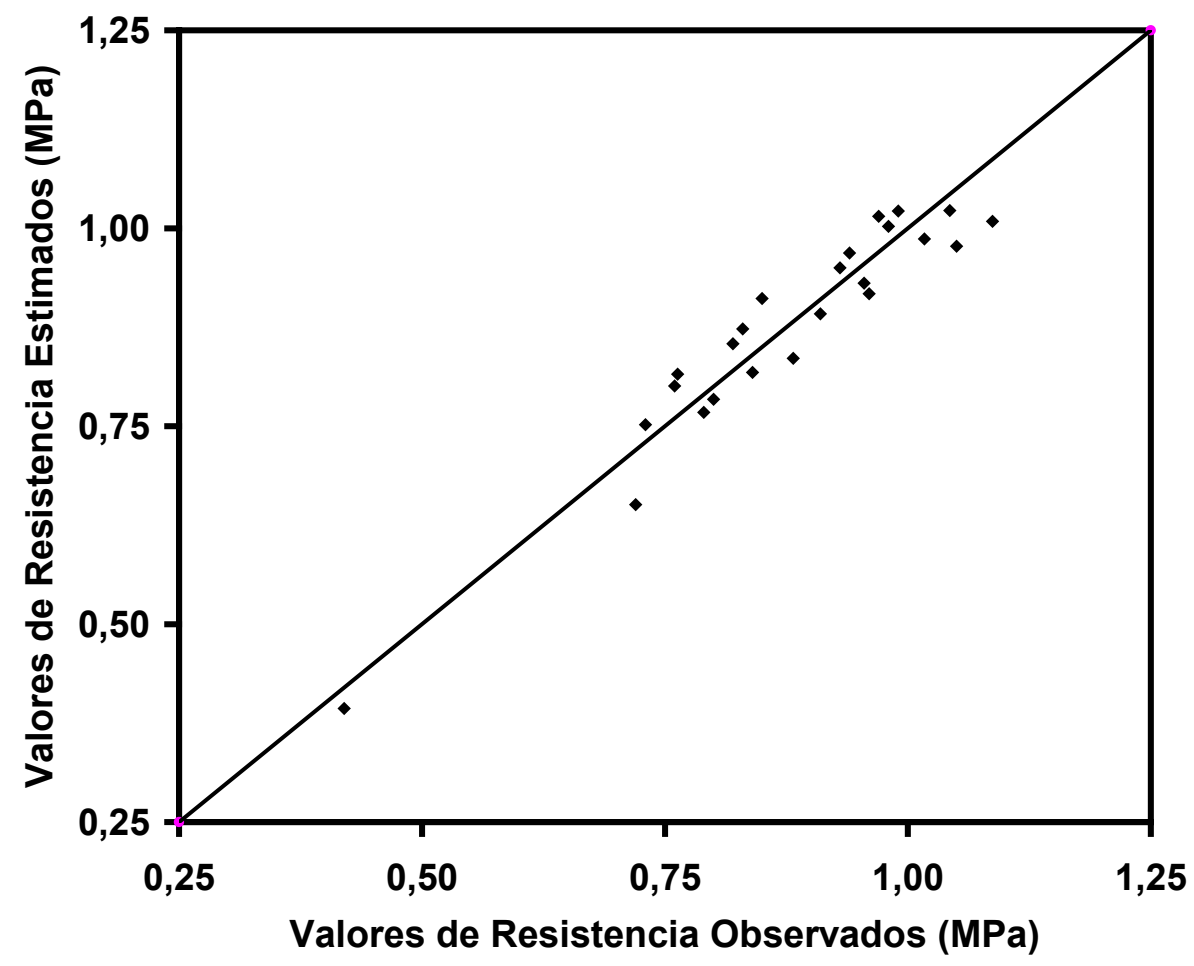

Figura 4. Valores de la resistencia del suelo a la penetración estimados, en función de los observados durante el período húmedo. 


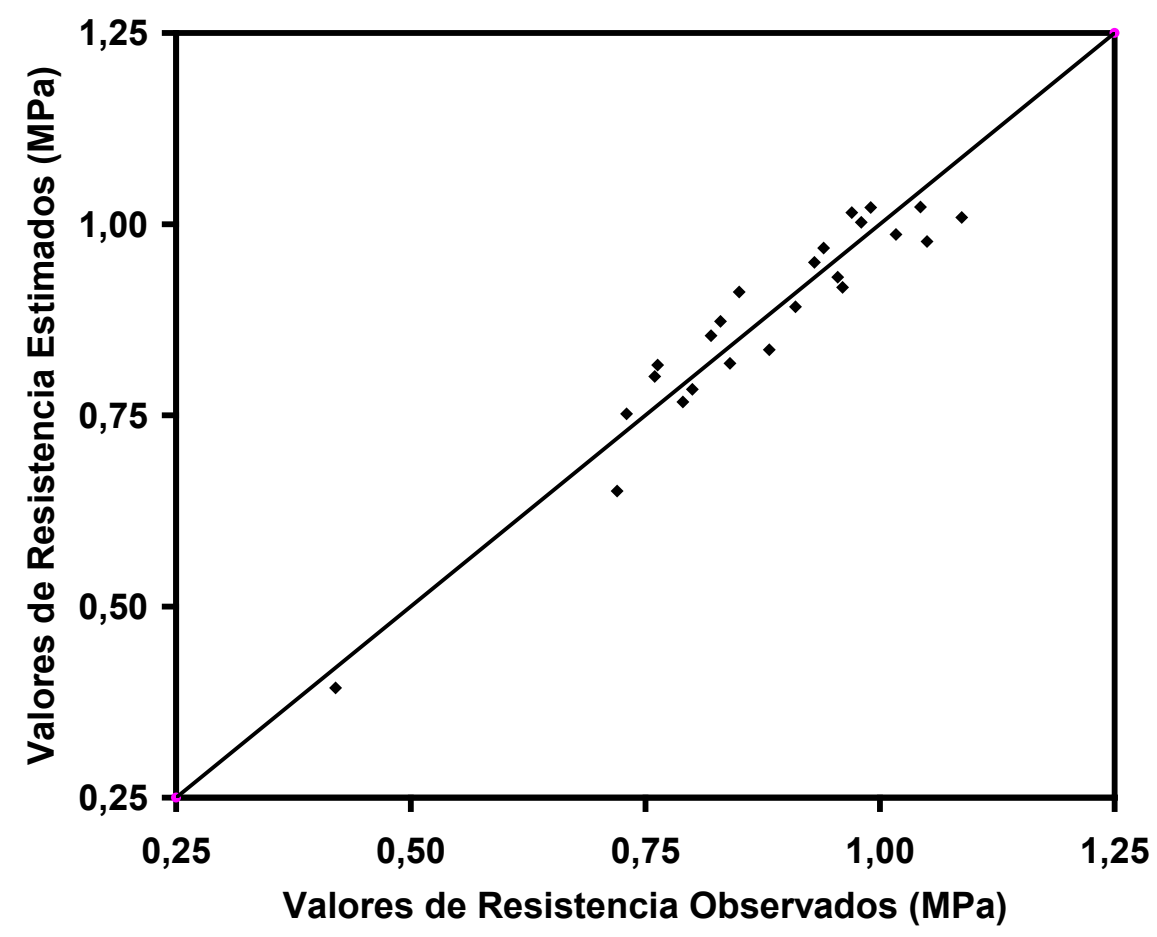

Figura 5. Valores de la resistencia del suelo a la penetración estimados, en función de los observados durante el período intermediario.

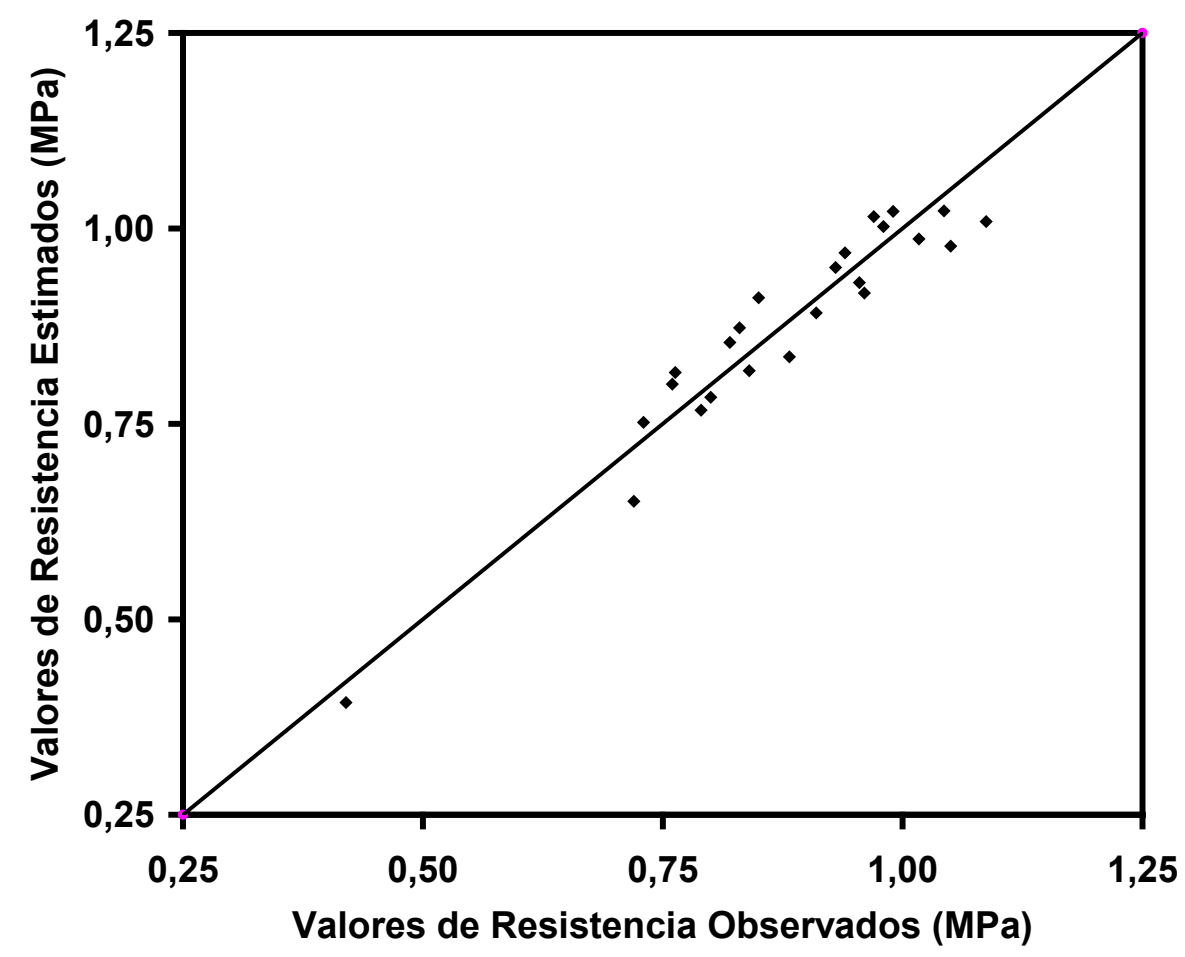

Figure 6. Valores de la resistencia del suelo a la penetración estimados, en función de los observados durante el período seco. 
En las Figuras 7, 8 y 9 se presentan los gráficos de los residuos para la resistencia del suelo a la penetración, de acuerdo con el período del año. Estos gráficos facilitan la inferencia respecto de alguna probable relación entre los residuos del referido atributo del suelo correspondiente a cada período del año. Con base en esas Figuras se deduce que no hay relación entre los residuos y el contenido de agua en el suelo, correspondiente a cada período del año, una vez que los puntos están distribuidos aleatoriamente encima y debajo del eje horizontal y no existen evidencias de algún padrón de dependencia funcional. También, de acuerdo con las Figuras 7, 8 y 9 se puede verificar que en el período húmedo, tanto la precisión cuanto la exactitud en las mediciones, fueron superiores, pues la dispersión y la magnitud de los residuos fueron inferiores.

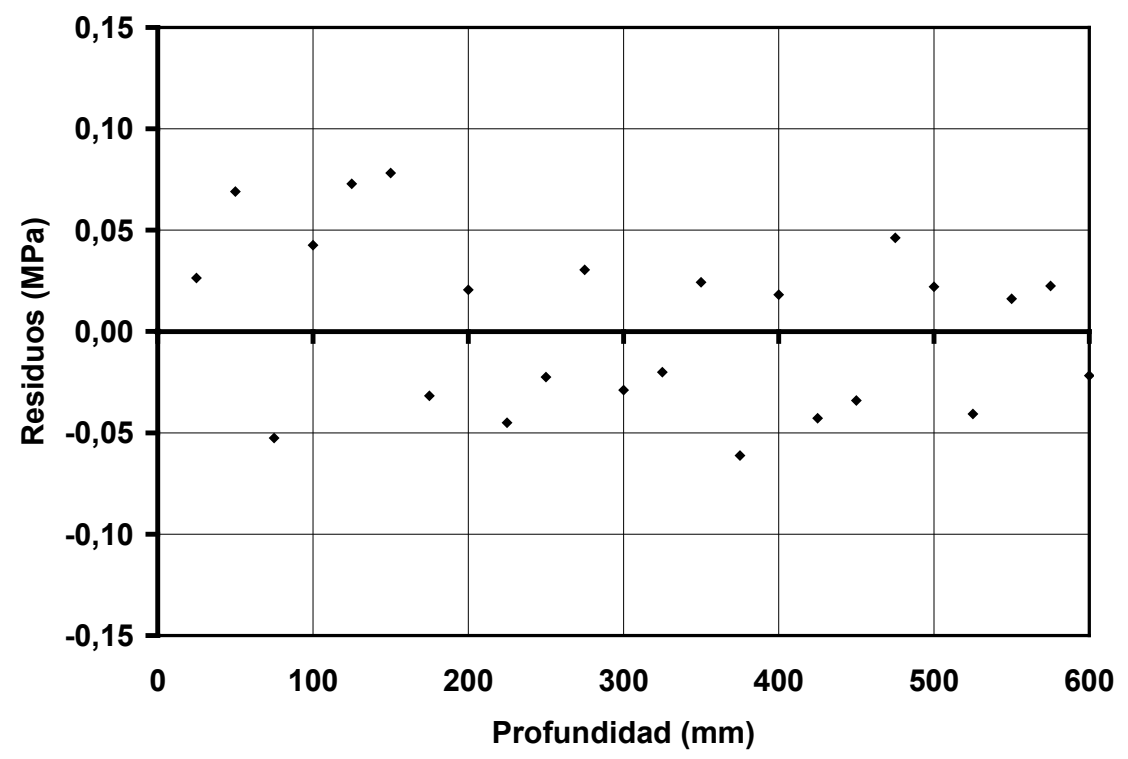

Figura 7. Gráfico de los residuos para la resistencia del suelo a la penetración evaluada durante el periodo húmedo.

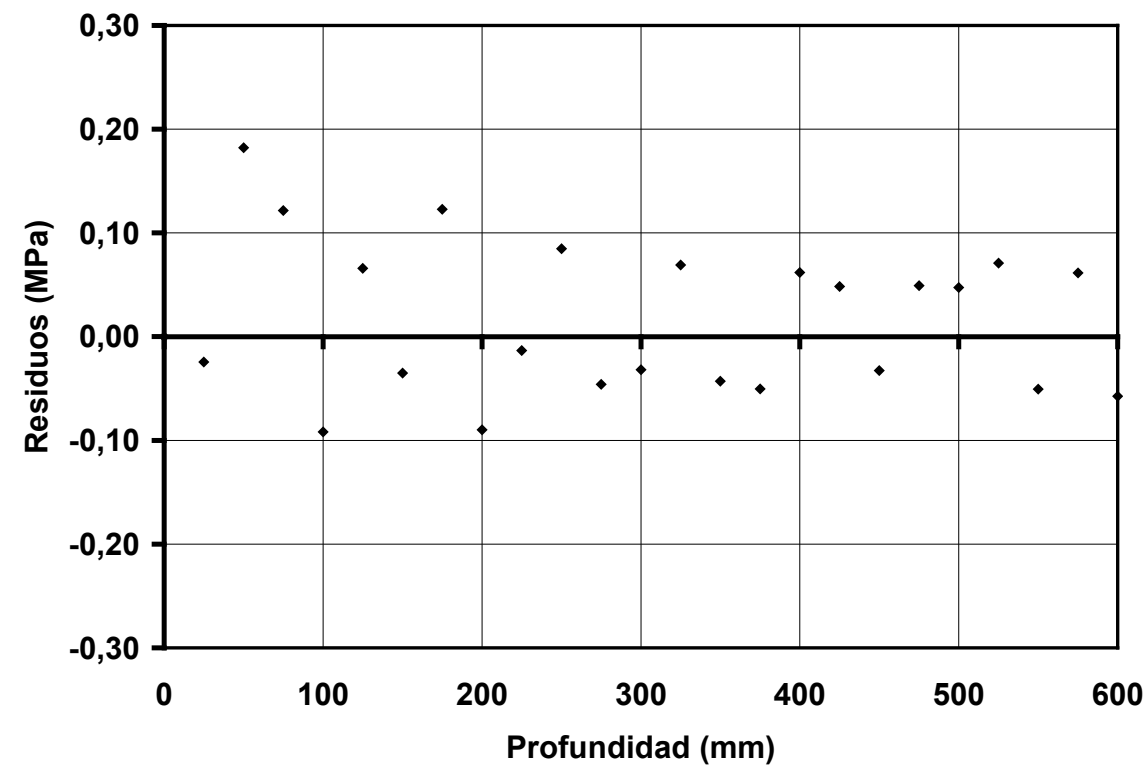

Figura 8. Gráfico de los residuos para la resistencia del suelo a la penetración evaluada durante el periodo intermedio. 


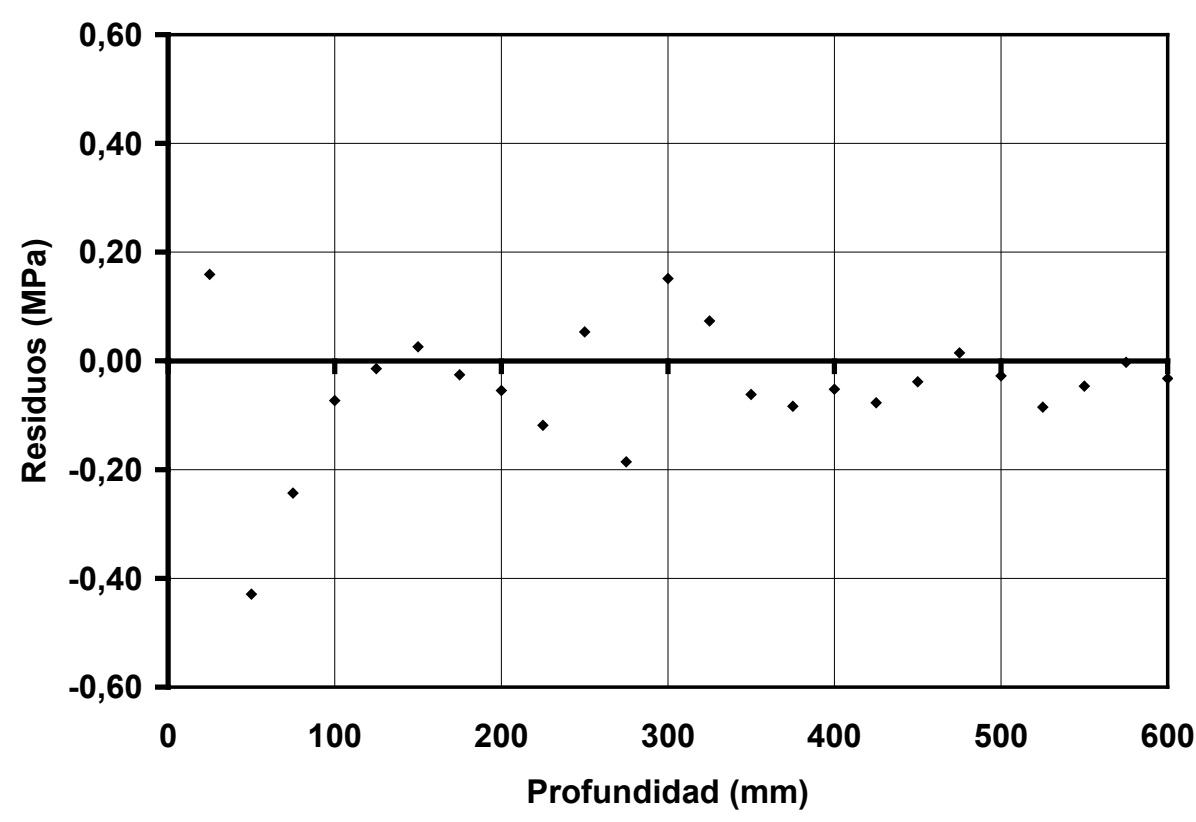

Figura 9. Gráfico de los residuos para la resistencia del suelo a la penetración evaluada durante el periodo seco.

La exigencia de normalidad de los residuos correspondiente a los modelos desarrollados puede ser avaluada a partir de su distribución de frecuencia (Figuras 10, 11 y 12). De acuerdo con esas Figuras se puede afirmar que los valores de los residuos presentaron una distribución normal con adecuada simetría, lo que fue comprobado analíticamente por las pruebas de Kolmogorov-Smirnov y Lilliefors en nivel de $5 \%$ de probabilidad. También, en las referidas Figuras se puede verificar que aproximadamente $80 \%$ de 1 os residuos estimados para el período húmedo se encuentran entre $-0,05$ y $0,05 \mathrm{MPa}$, sin embargo para los otros dos períodos no fue superior a $45 \%$. Estos resultados indican una mayor exactitud en las mediciones realizadas durante el período húmedo.

En esta investigación se consideró necesario verificar la independencia de los residuos, en función del tiempo, pues los datos fueron coleccionados en tres períodos del año. Se sabe que cuando los residuos a lo largo del tiempo siguen alguna tendencia puede existir un padrón denominado auto correlación y la validad del modelo estadístico propuesto puede estar comprometida. Para detectar si hubo auto correlación en el conjunto de datos analizados fue elaborado el gráfico de residuos, en orden secuencial del tiempo (Figura 13).

En la Figura 13 se observa que los residuos, en función del tiempo de colección de los datos no presentaron cualquier evidencia de un padrón, es decir, los residuos se distribuyeron aleatoriamente encima y debajo del eje horizontal, independientemente del período del año. Esa distribución es considerada por Montgomery et al. (2004) y Levine et al. (2005) como el comportamiento ideal para indicar ausencia de auto correlación. La confirmación analítica de que no hubo auto correlación fue basada en la estadística de Durbin-Watson para una variable independiente y 25 observaciones, cuyos resultados se presentan en el Cuadro 4. Como la estadística calculada es mayor que los limites superiores para los dos niveles de significancia, podemos afirmar que los residuos son independientes (Cuadro 4). 


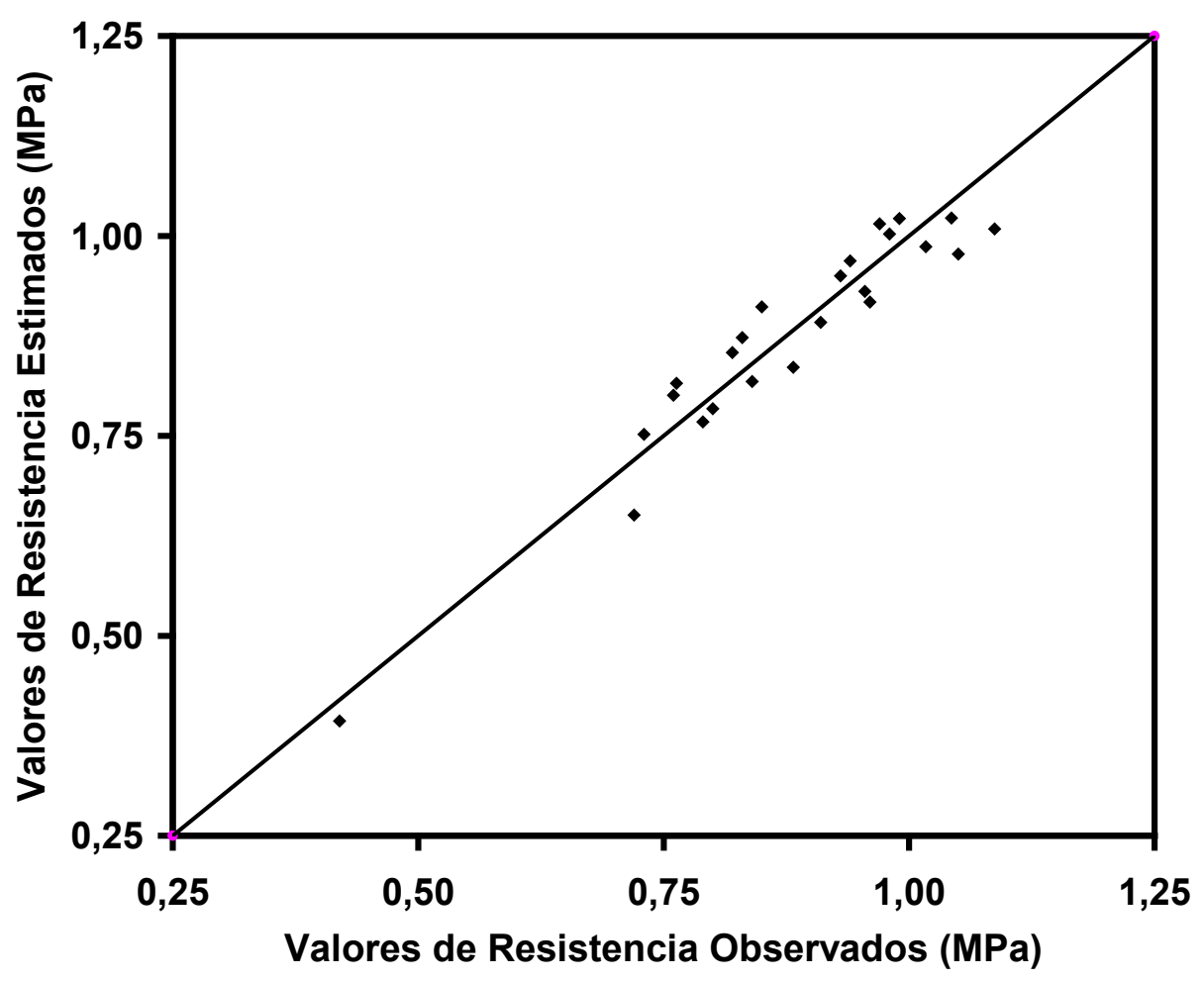

Figura 10. Histograma de residuos correspondientes al período húmedo.

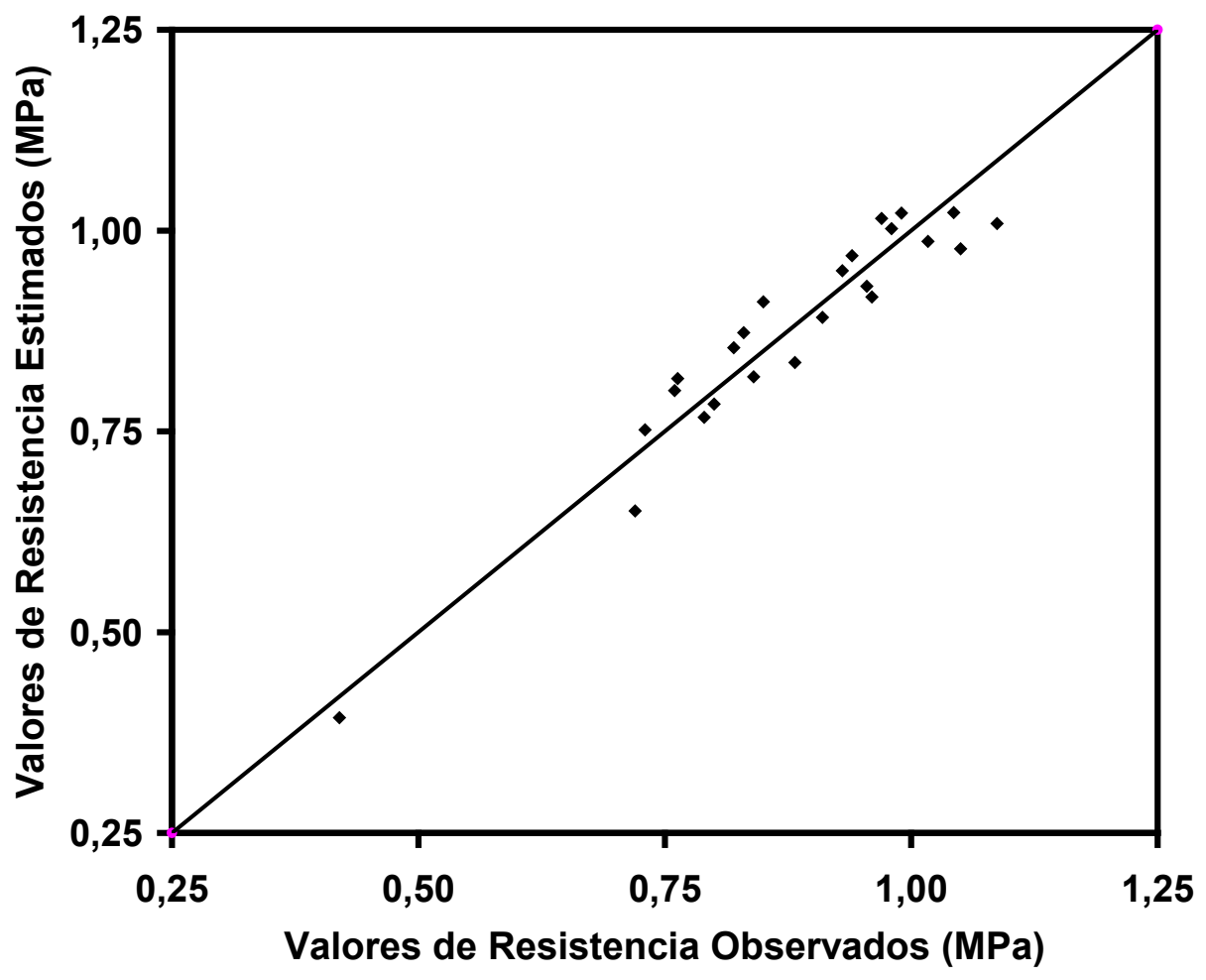

Figura 11. Histograma de residuos correspondientes al período intermediario. 


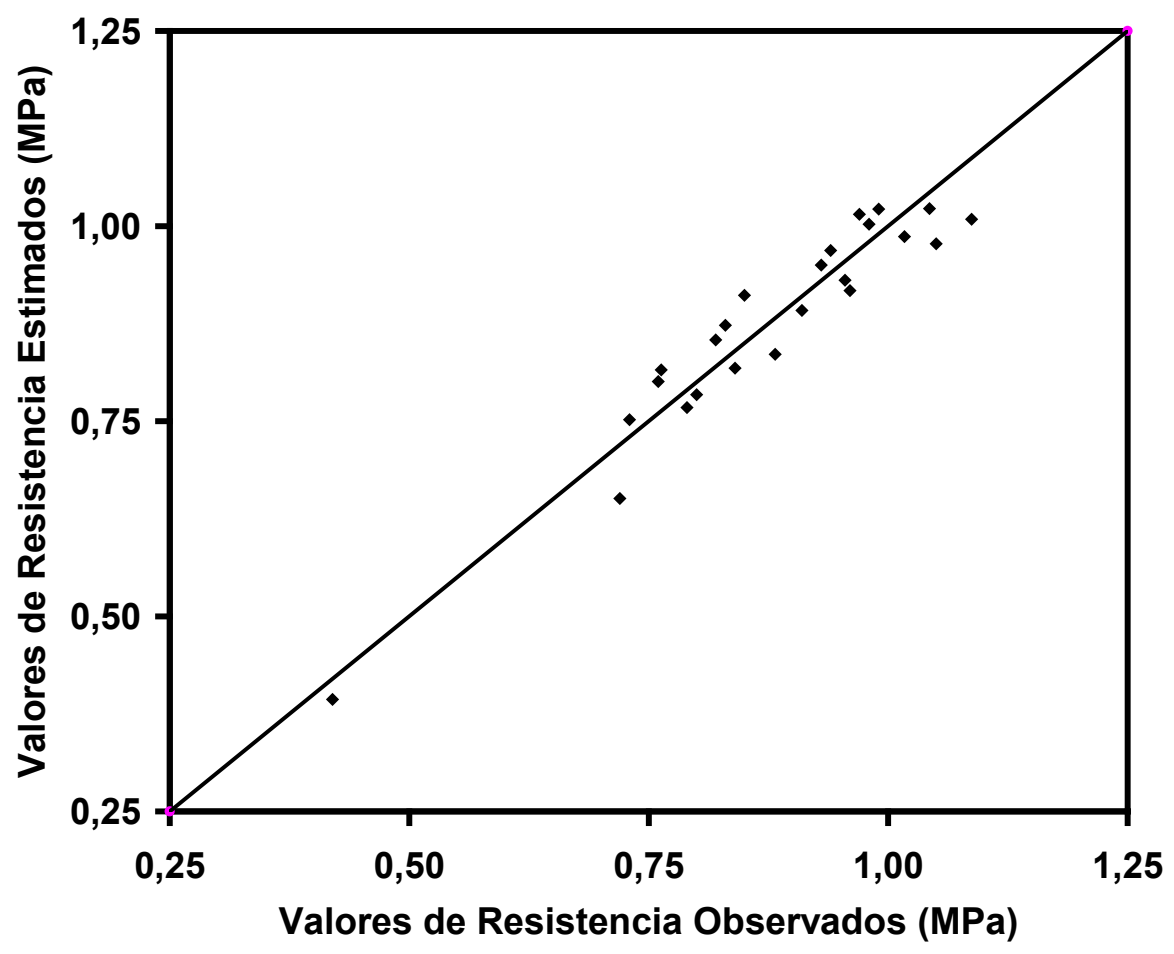

Figura 12. Histograma de residuos correspondientes al período seco.

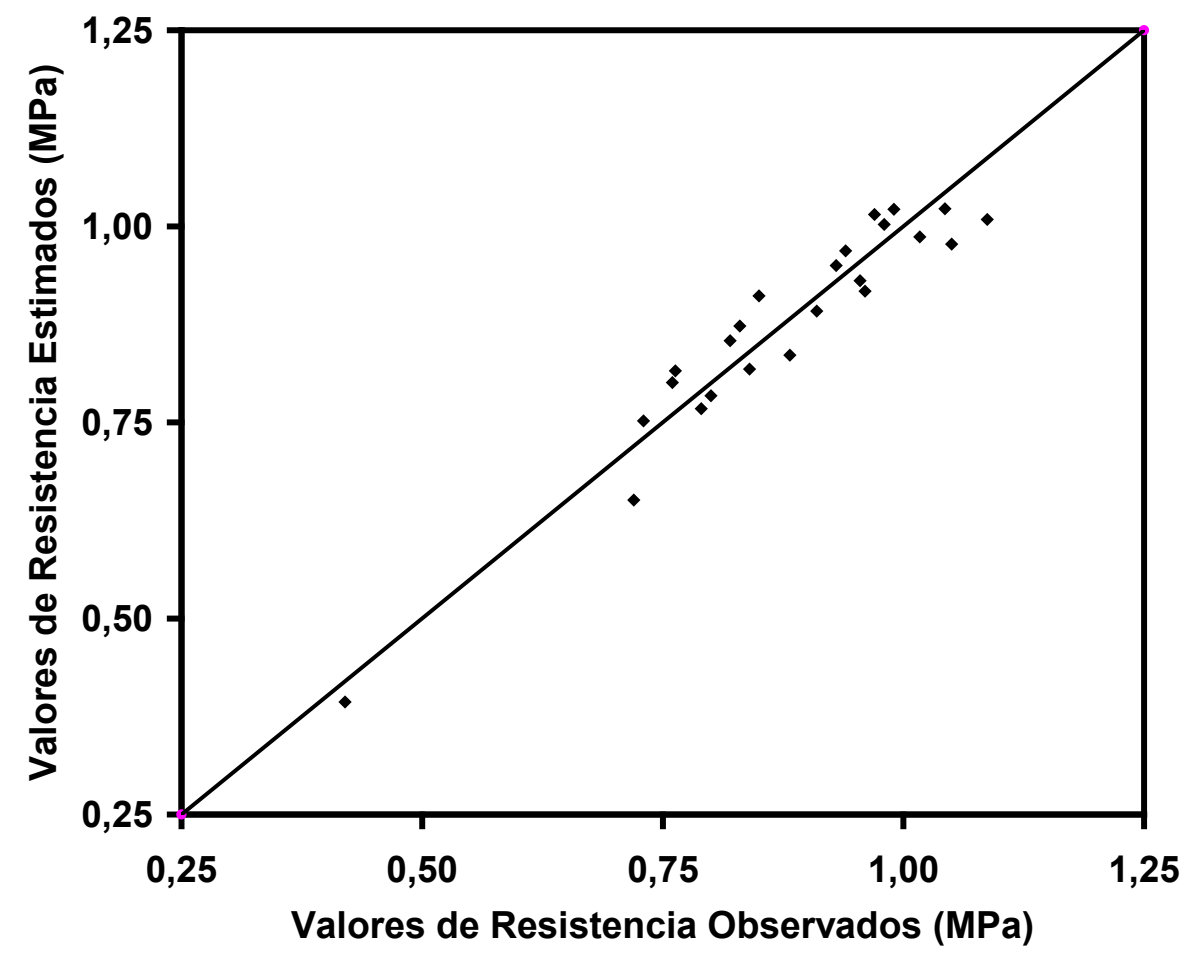

Figura 13. Gráfico de los residuos para la resistencia del suelo a la penetración, en función del contenido de humedad en cada período del año. 
Cuadro 4. Resultados de la estadística de Durbin-Watson para los tres períodos del año.

\begin{tabular}{|c|c|c|c|c|c|c|}
\hline \multirow{4}{*}{ Período del año } & \multicolumn{5}{|c|}{ Estadística de Durbin-Watson } & \multirow{4}{*}{ Auto correlación } \\
\hline & \multirow{3}{*}{ Calculada } & \multicolumn{4}{|c|}{ Limites tabulados } & \\
\hline & & \multicolumn{2}{|c|}{$\alpha=5 \%$} & \multicolumn{2}{|c|}{$\alpha=1 \%$} & \\
\hline & & $\begin{array}{c}\text { Inferior } \\
\left(\mathrm{d}_{\mathrm{I}}\right)\end{array}$ & $\begin{array}{l}\text { Superior } \\
\left(d_{\mathrm{s}}\right)\end{array}$ & $\begin{array}{c}\text { Inferior } \\
\left(\mathrm{d}_{\mathrm{I}}\right)\end{array}$ & $\begin{array}{c}\text { Superior } \\
\left(d_{\mathrm{S}}\right)\end{array}$ & \\
\hline Húmedo & 2,08 & \multirow{3}{*}{1,29} & \multirow{3}{*}{1,45} & \multirow{3}{*}{1,05} & \multirow{3}{*}{1,21} & No existe \\
\hline Intermedio & 1,93 & & & & & No existe \\
\hline Seco & 1,86 & & & & & No existe \\
\hline
\end{tabular}

Obs. $\alpha$ : Nivel de probabilidad

En este trabajo se verifico que todas las premisas de la regresión fueron satisfechas y que el modelo estadístico correspondiente al período húmedo fue el más preciso. Los resultados analíticos y gráficos obtenidos permiten afirmar que las dependencias funcionales propuestas pueden ser apropiadas para estimar la resistencia del suelo a la penetración, en función de la profundidad, considerándose el período del año. Esos modelos pueden facilitar el planeamiento del implemento adecuado para el preparo del suelo, pues podemos estimar previamente la resistencia del suelo a la penetración para una profundidad requerida.

\section{CONCLUSIONES}

De acuerdo con los resultados obtenidos, se concluye que:

- Los modelos desarrollados fueron apropiados para describir analíticamente la resistencia del suelo a la penetración, en función de la profundidad considerándose el período del año;

- Las ecuaciones empíricas obtenidas presentaron adecuada confiabilidad en las predicciones, lo que justifica su aplicación;

- Las dependencias funcionales propuestas pueden ser una herramienta útil y práctica para inferir sobre la compactación del suelo y definir el implemento necesario en el manejo del mismo.

\section{REFERENCIAS BIBLIOGRAFICAS}

ACOSTA, J.J.B. Índice de cone, capacidade de suporte de carga e teor de água de quatro solos da fazenda Lageado, Botucatu/sp: correlações e mapas de isocompactação. 2008. 145f. Tese (Doutorado em Agronomia). Universidade Estadual Paulista "JÚLIO DE MESQUITA FILHO", Faculdade de Ciências Agronômicas, Botucatu, 2008.

ALVES, S.M.F.; SERAPHIN, J.C. Coeficiente de heterogeneidade do solo e tamanho de parcela. Pesquisa Agropecuária Brasileira, Brasília, v.39, n.2, p.105-111, 2004.

AMERICAN SOCIETY OF AGRICULTURAL AND BIOLOGICAL ENGINEERS - ASABE. Soil cone penetrometer. St. Joseph: ASABE, ASAE Standard S313.3., p.902-904, 2006.

ASSIS, R.L.; LANÇAS, K.P. Avaliação dos atributos físicos de um nitossolo vermelho distroférrico sob sistema plantio direto, preparo convencional e mata nativa. Revista Brasileira de Ciência do Solo, Viçosa, v.29, n.4, p.515-522, 2005.

ASSIS, R.L.; LAZARINI, G.D.; LANÇAS, K.P.; CARGNELUTTI FILHO, A. Avaliação da resistência do solo à penetração em diferentes solos com a variação do teor de água. Revista Engenharia Agrícola, Jaboticabal, v.29, n.4, p.558-568, out./dez. 2009. 
BEUTLER,A.N.; CENTURION, J. F. Compactação do solo no desenvolvimento radicular e na produtividade de soja. Pesquisa Agropecuária Brasileira, Brasília, v.39, n.6, p.581-8, 2004.

BEUTLER, A.N.; CENTURION, J.F.; SILVA, A.P.; BARBOSA, J.C. Intervalo hídrico ótimo e produtividade de cultivares de soja. Revista Brasileira de Engenharia Agrícola e Ambiental, Campina Grande, v.10, n.3, p.639-645, 2006.

BONNIN, J.J.; MIRÁS-ÁVALOS, J.M.; LANÇAS, K.P.; GONZÁLEZ, A.P.; VIEIRA, S.R. Spatial variability of soil penetration resistance influenced by season of sampling. Bragantia, Campinas, v.69, Suplemento, p.163-173, 2010.

CARVALHO, G.J.; CARVALHO, M.P.;FREDDI, O.S.; MARTINS, M.V. Correlação da produtividade do feijão com a resistência à penetração do solo sob plantio direto. Revista Brasileira de Engenharia Agrícola e Ambiental, Campina Grande, v.10, n.3, p.765-771, 2006.

CUNHA, J.P.A.R.; VIEIRA, L.B.; MAGALHÃES, A.C. Resistência mecânica do solo à penetração sob diferentes densidades e teores de água. Engenharia na Agricultura, Viçosa, v.10, n.1-4, p.1-7, jan.-dez. 2002.

CUNHA, J.P.A.R.; CARVALHO JÚNIOR, P.C.; SOUZA, J.V.; BORGES, E.N.; REIS, E.F. Compactação do solo sob sistemas de manejo convencionais e conservacionistas. Revista Engenharia na Agricultura, Viçosa, v.17, n.2, p.155-162, 2009.

EMBRAPA - Empresa Brasileira de Pesquisa Agropecuária. Centro Nacional de Pesquisa dos solos. Sistema brasileiro de classificação dos solos. Brasília, 1999. 412p.

FABRIZZI, K.P.; GARCÍA, F.O.; COSTA, J.L.; PICONE, L.I. Soil water dynamics, physical properties and corn wheat response to minimum and no-tillage systems in the southern Pampas of Argentina. Soil \& Tillage Research, v.81, n.1, p.57-69, jan.-fev. 2005.

GREGO, C.R.; VIEIRA, S.R. Variabilidade espacial de propriedades físicas do solo em uma parcela experimental. Revista Brasileira de Ciência do Solo, Viçosa, v.29, n.2, p.169-177, 2005.

KÖPPEN, W.; GEIGER, R. Klima der Erde. Gotha: Verlag Justus Perthes. 1928.

LEVINE, D.M.; STEPHAN, D.; KREHBIEL, T.C.; BERENSON, M.L. Estatística - Teoría e aplicações usando-se o Microsoft EXCEL em português. Rio de Janeiro: Livros Técnicos e Científicos Editora S.A., 2005. 819p.

MOLIN, J.P.; MAGALHÃES, R.P.; FAULIN, G.D. Análise espacial da ocorrência do índice de cone em área sob semeadura direta e sua relação com fatores do solo. Engenharia Agrícola, Jaboticabal, v.26, n.2, p.442-452, maio/ago, 2006.

MONTGOMERY, D. C.; RUNGER, G. C.; HUBELE, N. F. Estatística aplicada à engenharia. Rio de Janeiro: LTC, 2004. 335 p.

PÉREZ, M. Rendimiento de un tractor agrícola en función del sistema de labranza y la carga. I. Características de la tracción. Revista Bioagro, Barquisimeto, v.18, n.1, p.49-55, 2006.

SANTOS, P.A.; CARVALHO, M.P.; FREDDI, O.S.; KITAMURA, A.E.; FREITAG, E.E.; VANZELA, L.S. Correlação linear e espacial entre o rendimento de grãos do feijoeiro e a resistência mecânica à penetração em um Latossolo Vermelho distrófico. Revista Brasileira de Ciência do Solo, Viçosa, v.29, n.2, p.287-295, 2005.

TORMENA, C.A.; BARBOSA, M.C.; COSTA, A.C.S.; GONÇALVES, A.C.A. Densidade, porosidade e resistência à penetração em Latossolo cultivado sob diferentes sistemas de preparo do solo. Scientia Agrícola, Piracicaba, v.59, n.4, p.795-801, 2002.

WEIRICH NETO, P.H.; BORGHI, E.; SVERZUT, C.B.; MANTOVANI, E.C.; GOMIDE, R.L.; NEWES, W.L.C. Análise multivariada da resistência do solo à penetração sob plantio direto. Ciência Rural, Santa Maria, v.36, n.4, p.11861192, jul-ago, 2006.

WEISBERG, S. Applied Linear Regression. Wiley: A John Wiley \& Sons, Inc., 2005. 330p. 